\title{
Narrow band and energy selectable plasma cathode for multistage laser wakefield acceleration
}

\author{
Y. Sakai, ${ }^{1,2, \uparrow}$ N. Pathak, ${ }^{1,2}$ Z. Jin, ${ }^{2}$ A. Zhidkov, ${ }^{1,2}$ K. Sueda, ${ }^{2}$ H. Toran, ${ }^{1}$ Y. Tanizawa, ${ }^{1}$ \\ T. Otsuka, ${ }^{1,2, *}$ J. Ogino, ${ }^{3}$ H. Nakamura, ${ }^{1}$ R. Kodama, ${ }^{1,3}$ and T. Hosokai ${ }^{1,2}$ \\ ${ }^{1}$ Graduate School of Engineering, Osaka University, 2-1 Yamada-oka, Suita, Osaka 565-0871, Japan \\ ${ }^{2}$ Laser Accelerator R\&D Team, Innovative Light Sources Division, RIKEN SPring-8 Center, 1-1-1, Kouto, \\ Sayo-cho, Sayo-gun, Hyogo 679-5148 Japan \\ ${ }^{3}$ Institute of Laser Engineering, Osaka University, 2-1 Yamada-oka, Suita, Osaka 565-0871, Japan
}

(Received 13 July 2018; published 3 October 2018)

\begin{abstract}
Stable, with a narrow energy band $<3 \%$, and energy selectable, from 1 to $40 \mathrm{MeV}$, electron beams with charges $\sim 1 \mathrm{pC}$ are produced from broadband electron bunches accelerated in the wakefield of femtosecond laser pulses with pulse energy $\sim 0.4 \mathrm{~J}$. The beams are extracted with use of energy filtering based on a compact and controllable pulse-driven solenoid. Such a plasma cathode based on laser wakefield acceleration can be used as an injector for the multistage laser wakefield electron acceleration as well as for ultrafast electron imaging systems.
\end{abstract}

DOI: 10.1103/PhysRevAccelBeams.21.101301

\section{INTRODUCTION}

In the last decade, laser wake field acceleration (LWFA) of electrons by femtosecond laser pulses is being evolved from theory to technology [1-5]. LWFA was mostly oriented for high-energy accelerators due to their ultrahigh electric field gradients. Electron energies near $4.2 \mathrm{GeV}$ have been already achieved with the use of peta watt class laser pulses [6]. On the other hand, having short bunch durations, low emittance, and being potentially jitter-free, LWFA electron beams are considered a framework for ultrafast electron and x-ray imaging [7-9]. In the context of LWFA several techniques based on gas jets, gas-cell, and preformed channels have been proposed and tested experimentally [10-18].

It is clear that parameters of the acceleration field should not be changed during an acceleration process. However, during laser pulse propagation in a long distance its parameters usually essentially change. It happens because of pulse depletion, sensitivity of dephasing length to pulse parameters, various instabilities such as self-focusing, hosing, and others $[5,11,12]$. As a result, achieving

\footnotetext{
*Present address: Department of Engineering, Utsunomiya University, 350 Minemachi, Utsunomiya 321-8505, Japan.

Present address: Graduate School of Engineering, Osaka University, 2-1 Yamada-oka, Suita, Osaka 565-0871, Japan. y.sakai@ppc.osaka-u.ac.jp

Published by the American Physical Society under the terms of the Creative Commons Attribution 4.0 International license. Further distribution of this work must maintain attribution to the author(s) and the published article's title, journal citation, and DOI.
}

necessary controllability and reproducibility of electron acceleration in the wakefield of such a pulse become difficult. One partial solution of this problem is to shorten the acceleration length in a single stage and use the multistage acceleration scheme [15,18]. The multistage acceleration allows also the use of different plasma parameters in each stage that makes the process of LWFA quite flexible.

In the multistage LWFA, the electron beam injection into the acceleration phase of the laser wakefield becomes a critical process. Roughly, injected electrons must have velocity exceeding the group velocity of a laser pulse in plasma: $v_{\mathrm{gr}} \approx \mathrm{c} \sqrt{1-N_{e} / a_{0} N_{\mathrm{cr}}}$, where $c$ is the speed of light, $N_{e}$ is the electron density in plasma, $N_{\mathrm{cr}}=$ $m_{e}(\omega / e)^{2} / 4 \pi$ is the critical density for laser pulse with frequency $\omega, a_{0}$ is the normalized vector potential of a laser pulse, and $m_{e}$ is the electron mass [19]. An injected electron beam has to have energy $\varepsilon_{i} \approx \mathrm{m} c^{2} \sqrt{a_{0} N_{\mathrm{cr}} / N_{e}}$. For Ti-Sapphire laser pulses with wavelength $\lambda=800 \mathrm{~nm}$, the critical density is $N_{\mathrm{cr}}=1.74 \times 10^{21} \mathrm{~cm}^{-3}$. Therefore, for the injection of electrons in plasma with density $N_{e}=$ $2 \times 10^{18} \mathrm{~cm}^{-3}$ (as in Ref. [19]]) irradiated by a laser pulse with $a_{0} \sim 1$, the energy should exceed $\varepsilon_{i}=15 \mathrm{MeV}$. Therefore, electrons should be preaccelerated in the plasma cathode, which is apparently the first stage of the multistage LWFA. Since the spot size of a laser pulse with $a_{0} \sim 1$ is usually less than $100 \mu \mathrm{m}$ in diameter, the electron beams should have a quite small size for an efficient injection or coupling with the second booster stage. To satisfy all of these conditions, electron beams extracted from the plasma cathode should have a low beam divergence and should be stable and well controlled. Beam energy tuning is also a 
required parameter, which is necessary for efficient injection of the electrons in the acceleration phase of the wakefield.

It is well known that LWFA of electrons in a dense uniform plasma results in their broad, Maxwell-like distribution over energy [19] with an effective temperature $T_{\text {eff }}=\chi m_{e} c^{2} a_{0}$, where $\chi$ lays in the range from 1 to $\sim 10$ depending on plasma parameters. Tightly focused laser pulses with $a_{0} \sim 2-5$, therefore, can produce a considerable charge of electrons with energy from 1 to $70 \mathrm{MeV}$ with a low emittance $[20,21]$. On the other hand, by using a magnetic lens and a set of pin holes, one can extract a reasonable portion of such electrons with a quite narrow energy band. In this case the magnetic lens works as a linear energy filter. The filter is linear because it does not change the initial energy distribution of electrons. Upon controlling the characteristic of the magnetic lens one can tune the energy of extracted electrons in a broad range. In the present work, we investigate the characteristics of electron beams from a laser-plasma cathode based on LWFA using a pulse driven solenoid lens as an energy filter as well as a beam spatial collector. We also explore the applicability of this technique for the energy range below $10 \mathrm{MeV}$, which is important for the ultrafast electron diffraction imaging [22].

\section{EXPERIMENTAL SETUP}

Experimental setup is shown in Fig. 1. The experiment was carried out with the 20 TW-30 fs Ti:Sapphire laser system at Photon Pioneers Center, Osaka University, based on a chirped pulse amplification technique [23]. The pulse energy on a target was $450 \mathrm{~mJ}$ at its maximum and the pulse duration was $30 \mathrm{fs}$ (FWHM). The laser spot diameter was $8 \mu \mathrm{m}\left(1 / e^{2}\right)$ and can provide the maximal intensity
$I=2 \times 10^{19} \mathrm{~W} / \mathrm{cm}^{2}$. Considering the energy concentration of $50 \%$, the on-target intensity is estimated to be $1 \times 10^{19} \mathrm{~W} / \mathrm{cm}^{2}$, which corresponds to the normalized vector potential of $a_{0} \sim 3$. The central wavelength of the laser pulse is $\lambda=800 \mathrm{~nm}$. The contrast ratio between the main pulse and the nanosecond prepulse caused by the amplified spontaneous emission was set to be about $10^{-9}$. Picosecond contrast was of the order of $10^{-6}$ at $5 \mathrm{ps}$ prior to the main pulse.

The laser was focused on the front edge of a uniform helium gas-jet target produced by a supersonic slit nozzle, with a gold-coated off-axis parabolic mirror with $f / 3.5$ $(f=163 \mathrm{~mm})$. The thickness of the gas jet was $1.2 \mathrm{~mm}$. The stagnation pressure at the reservoir of the nozzle was set up to $4 \mathrm{MPa}$ with the gas density at the laser axis orders of $N=10^{19} \mathrm{~cm}^{-3}$ similarly to that in our previous experiment [17].

Spatial distribution of the ejected electron beams was measured by a phosphor screen (Mitsubishi Chemical Co. LTD, DRZ-High [24]). Two screens were located at $48 \mathrm{~cm}$ and $159 \mathrm{~cm}$ away from the gas-jet target $(\mathrm{T})$ as beam monitors (BM1 and BM2) as shown in Fig. 1. The phosphor screen is sensitive to high-energy particles and radiations, so that the front side of the screen was laminated with a $12 \mu \mathrm{m}$-thick aluminum foil to avoid exposure to the laser pulses, scattering lights, and low-energy electrons. The scintillating images on these phosphor screens made by the deposited electrons were recorded by 16 bit charged coupled device (CCD) cameras with imaging lenses from the backside of the screen.

Parameters of the pulse solenoid lens were chosen to provide necessary focus ability, with the focus spot within $2 \mathrm{~m}$ distance from the gas jet (T), for electron beams with

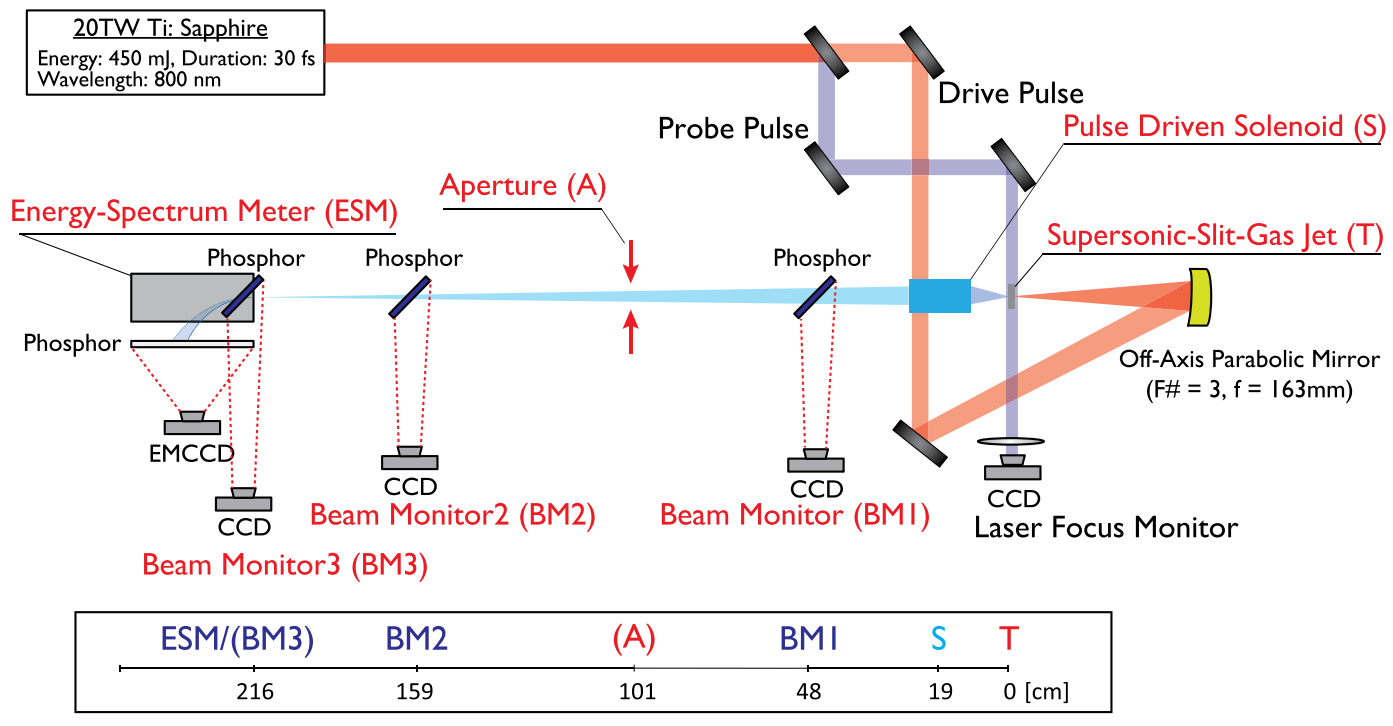

FIG. 1. Schematic diagram of experimental setup; the entrance position of the longitudinal direction (beam propagating direction) for each component is indicated in the bottom scale. T, S, BMs, A, and ESM indicate the positions of the gas target, solenoid, beam monitor, aperture, and energy spectrum meter, respectively. Beam monitors (BM1-3) and the aperture (A) are removable. 
energy ranging from 1 to $60 \mathrm{MeV}$. Required focusing magnetic strength can be estimated with a simplified model with the well-known thin-lens approximation for paraxial charged particle lay. For relativistic electron beams, we can get the relation $1 / f=\left(e B_{z} / 2 \gamma m_{e} v_{z}\right)^{2} l$, where $f$ is the focal length of the solenoid lens, $B_{z}$ is the longitudinal component of the solenoidal magnetic field, $\gamma$ is the Lorentz factor, $v_{z}$ is the electron longitude velocity, and $l$ is the length of the lens, respectively. For solenoid coils, the longitudinal magnetic field is derived by $B_{z}=k \mu_{0} n I$, here $\mu_{0}$ is the magnetic permeability in vacuum, $n$ is the number of turns per meter, $I$ is the drive current, and $k$ is Nagaoka's coefficient, respectively. If the geometrical configuration is determined, one can get the required drive current. Focus ability and required drive current for a $50 \mathrm{~mm}$ length, 3400 turns per meter $(170$ turns $/ 50 \mathrm{~mm})$, and $10 \mathrm{~mm}$ diameter solenoid lens configuration are shown in Fig. 2. Apparently, the system can focus electron beams of up to several tens of $\mathrm{MeV}$ within a several decimeter length by the high drive current of $\mathrm{kA}$ order.

Again, we used a pulse driven solenoid lens as a focusing and energy filtering system to extract electrons with a narrow energy band. The solenoid (S) was set at $19 \mathrm{~cm}$ away from the gas-jet target $(\mathrm{T})$. Figure 3 shows an equivalent circuit diagram of the pulse driven solenoid focus system and geometrical configuration of the solenoid lens in the inset. The solenoid coil was designed with $10 \mathrm{~mm}$ inner diameter and $50 \mathrm{~mm}$ length. It is able to transmit effectively electron bunches with $5 \mathrm{mrad}$ divergence. A thin wire laminated with polyamid (triple insulated wire TEX-E FURUKAWA [25]) able to withstand high voltage was use for the coil. It allowed an essential increase of driving magnetic field strength. The wire diameter of $0.6 \mathrm{~mm}$ was wrapped and wound on the

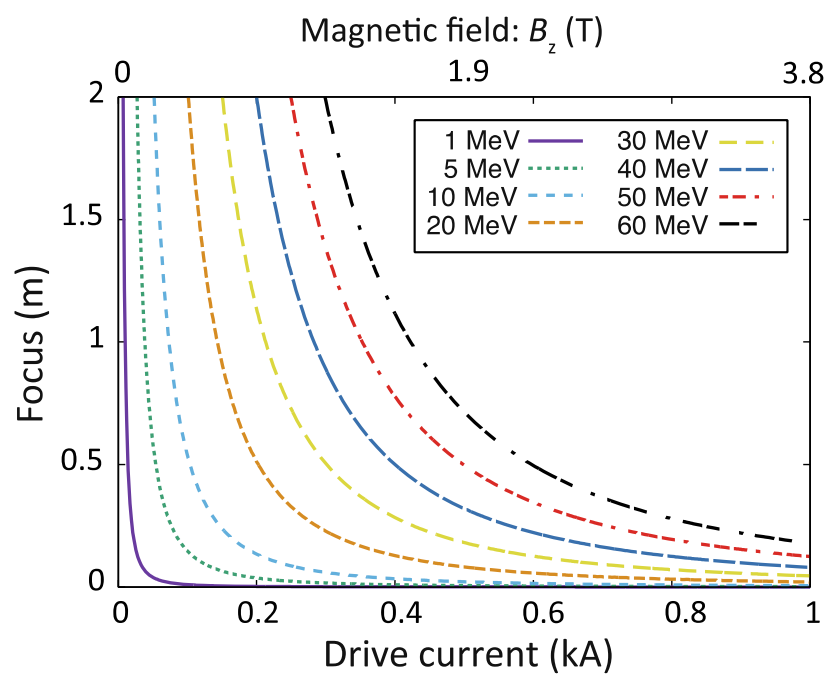

FIG. 2. Focus ability of a solenoid lens for electron beams with energy from 1 to $60 \mathrm{MeV}$ and the required drive current based on thin-lens approximation for $50 \mathrm{~mm}$ length and $10 \mathrm{~mm}$ inner diameter case.

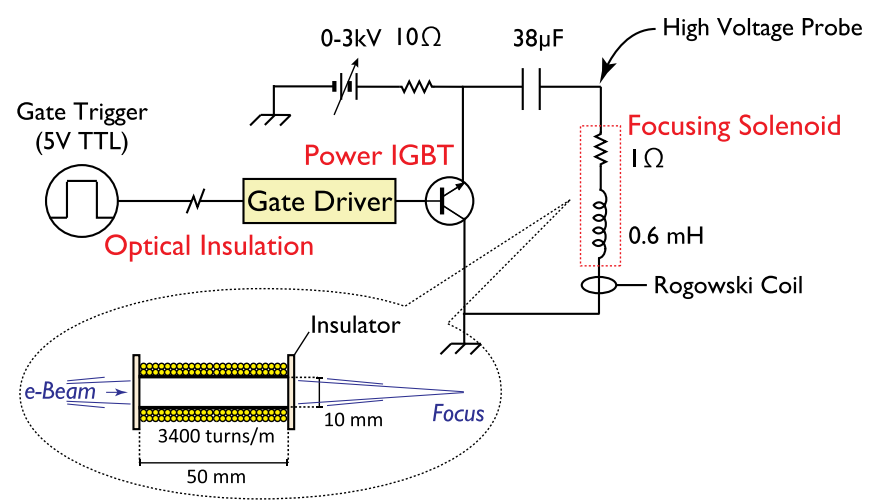

FIG. 3. Equivalent circuit diagram of the pulse-driven solenoid focus system and geometrical configuration of the solenoid lens (inset).

cylindrical insulator. The resistivity and inductance of the coil was $1 \Omega$ and $0.6 \mathrm{mH}$, respectively. For synchronization of the femtosecond duration electron bunches and the discharge current, the pulse driver was assembled with a low jitter solid-state switching device, power Insulated gate bipolar transistor (IGBT) (CM900HB-90H Mitsubishi electric [26]). The withstand voltage and current of the pulse driver were $4500 \mathrm{~V}$ and $900 \mathrm{~A}$, respectively. Figure 4 shows the typical output voltage and current waveforms of the pulse driver measured with a high voltage probe and a Rogowski coil as shown in Fig. 3. The charging voltage was set to $400 \mathrm{~V}$. As shown in Fig. 4, it can provide a drive current waveform with a rising time of $100 \mu \mathrm{s}$ and a half period of $200 \mu \mathrm{s}$. The flattop region of $15 \mu \mathrm{s}$ at $t=120 \mu \mathrm{s}$ is much longer than the electron bunch traveling time in the solenoid ( 170 ps). Therefore, the pulse magnetic field can be regarded as a constant field for traveling electron bunches. The electron beam energy was measured by a dipole permanent magnet energy spectrometer (ESM) located at $2 \mathrm{~m}$ downstream from the gas jet (T). The geometrical configuration of the dipoles is as follows: length is $700 \mathrm{~mm}$, width is $190 \mathrm{~mm}$, and gap between

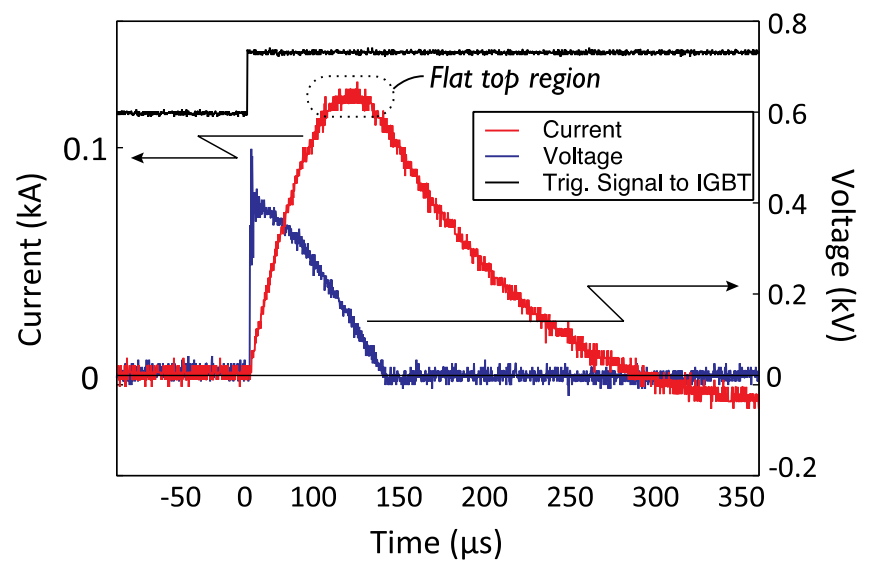

FIG. 4. Typical output voltage, drive current waveform of the device, and tiger pulse to IGBT. 


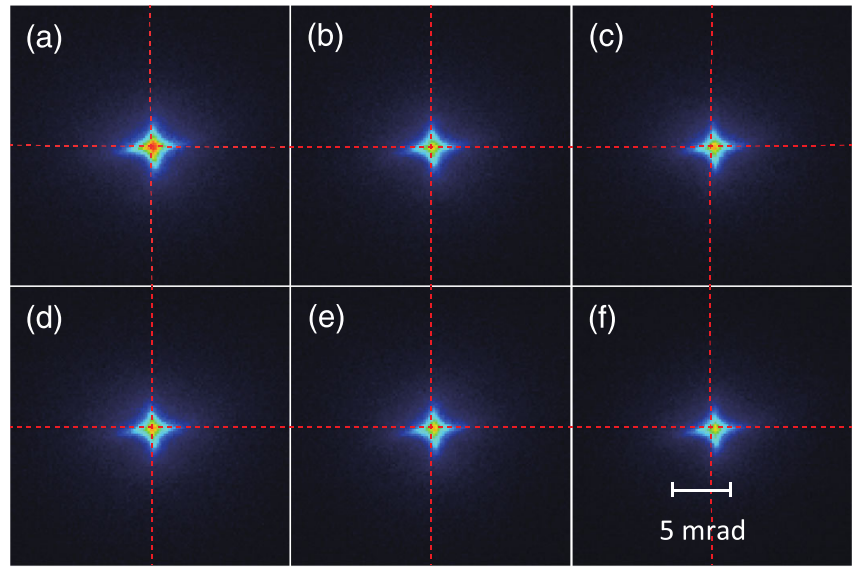

FIG. 5. Typical focusing electron beam profiles of 6 successive shots on the beam monitor screen at the $2 \mathrm{~m}$ (BM3) from the exit of the solenoid.
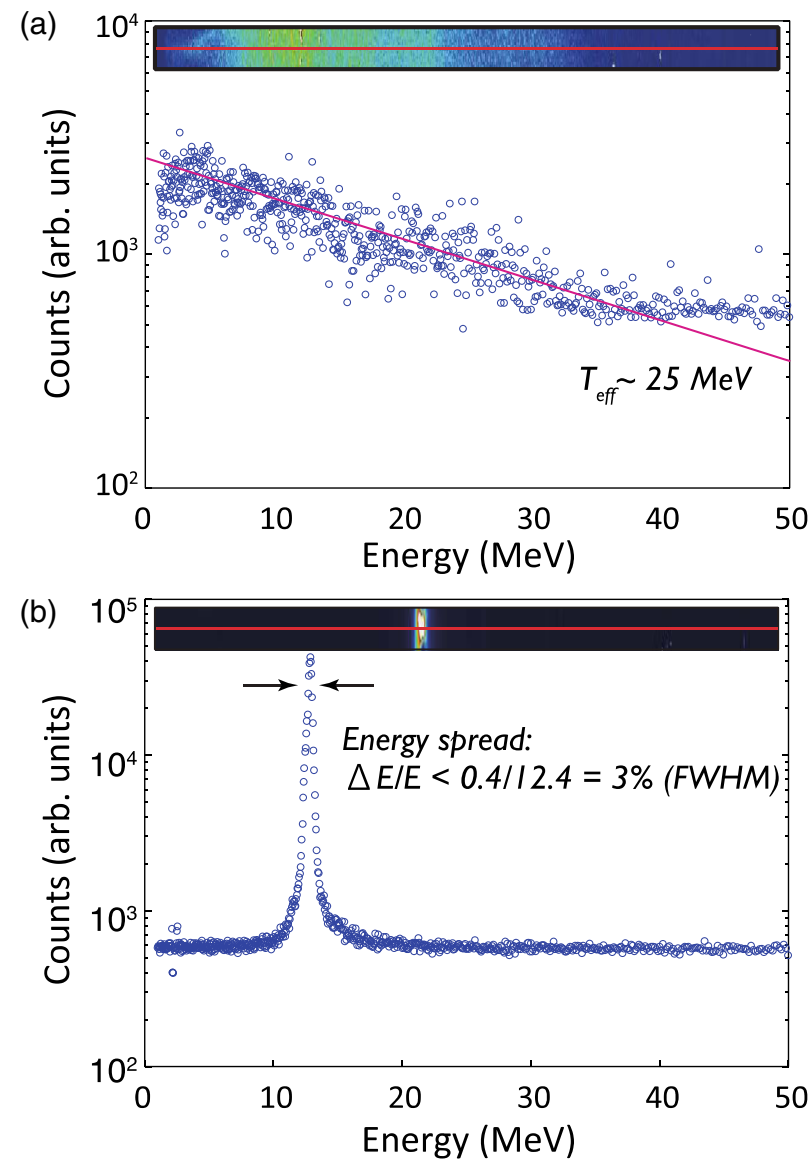

FIG. 6. Typical energy distributions of electron beam for (a) without energy selection with solenoid and (b) with solenoid focusing for electron central energy of $12.4 \mathrm{MeV}$. The corresponding images on an ESM screen are put with the spectrum. magnets is $30 \mathrm{~mm}$. A perpendicular slit of $5 \mathrm{~mm}$ in width was set in front of the entrance of the ESM. With this configuration the geometrical acceptance angle of the ESM is about $3 \mathrm{mrad}$.

\section{RESULTS AND DISCUSSION}

Images of focused beams with different energies were exposed on the phosphor screen and measured. Typical electron beam images on the screen at $2 \mathrm{~m}$ from the exit of the solenoid are shown in Fig. 5. These images were obtained by the phosphor screen set just behind the entrance aperture of ESM. As shown in Fig. 5, for the energy $\varepsilon \sim 10 \mathrm{MeV}$ the beam size was estimated to be smaller than $2 \mathrm{~mm}$ in FWHM. The pointing stability of the beams was estimated to be better than $0.5 \mathrm{mrad}$.

Results of energy filtering can be seen in Fig. 6 where we present images of the ESM screen and typical calibrated energy spectra on the axis for electron beams without (a) and with (b) the linear filter for energy $\varepsilon=12.4 \mathrm{MeV}$ detected on the image screen. Expectedly, the initial energy spectrum given in Fig. 6(a) is broad-banded with an effective temperature $T_{\text {eff }} \sim 25 \mathrm{MeV}$. This temperature essentially exceeds the temperature of the electron source at the exit of the gas jet. The reason is in dependency of beam divergence on electron energy: higher energy corresponds to smaller divergence. Therefore, the "effective" temperature of electrons reaching the image plate essentially increases, as indicated in Fig. 6(a). Because beams are transported through a drift-tube with the slit of $3 \mathrm{mrad}$ acceptance angle, one can observe a lack of low energy components, below $5 \mathrm{MeV}$.

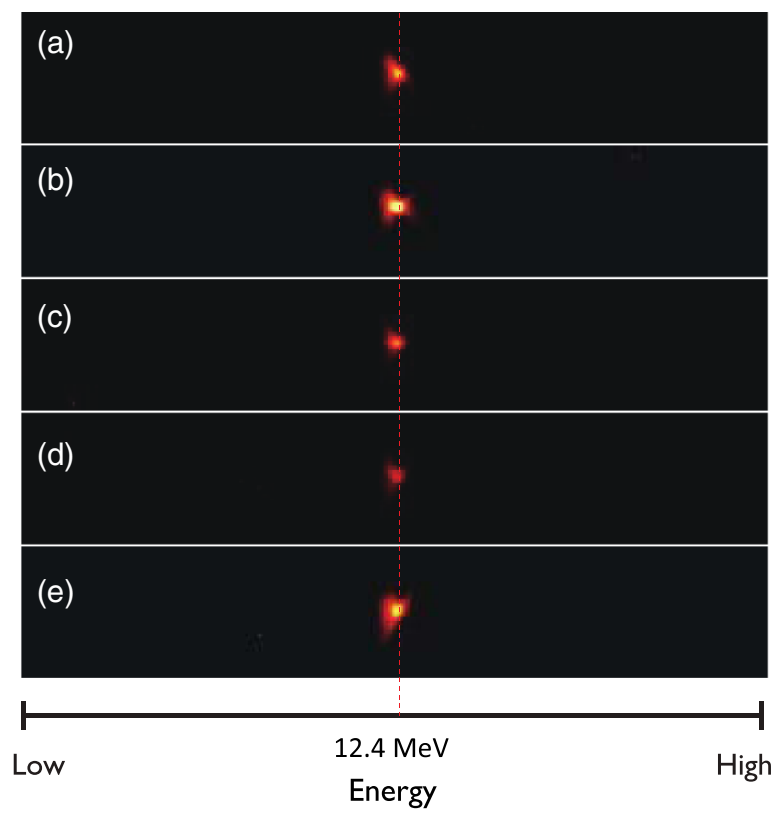

FIG. 7. Typical images of screen on an ESM for successive five shots (a)-(f). The vertical axis corresponds to spatial distribution of the electron beam. 


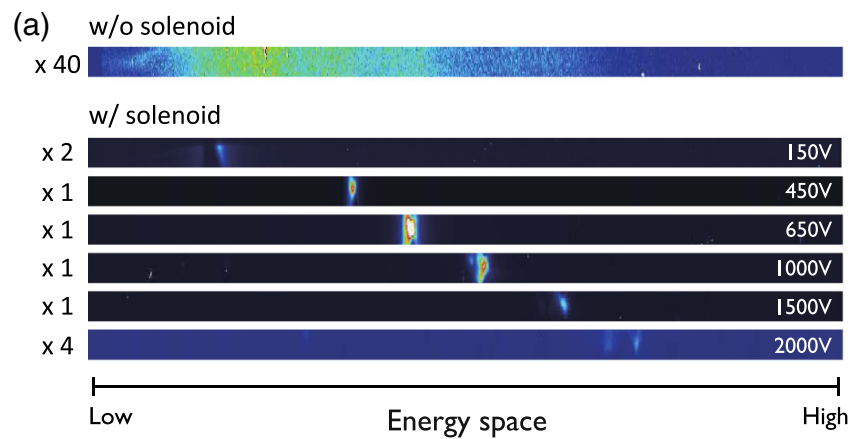

(b)

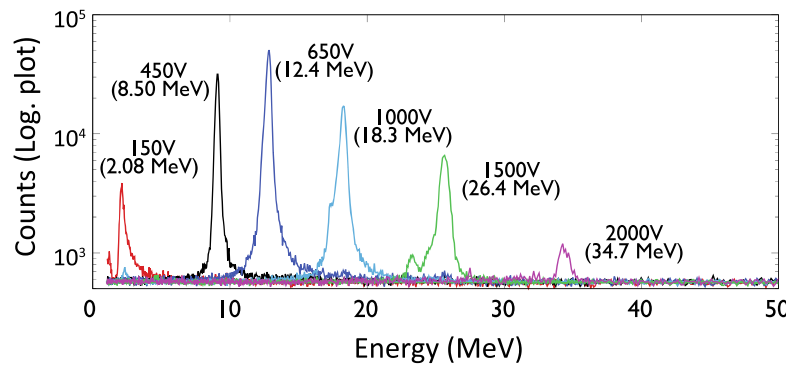

FIG. 8. Typical images of electron beam on an ESM screen (a) with applied voltages of the solenoid on the right side; the brightness of each image is adjusted for better presentation (the intensification is on the left side). The energy spectra on the axis corresponding to the images in (a) are shown in (b).

The pulse solenoid drastically improves the energy spectrum of the accelerated electrons on the axis as shown in Fig. 6(b). Beam average energy is $12.4 \mathrm{MeV}$ and energy spread is smaller than $400 \mathrm{keV}$ in FWHM. Note that the resolution of the ESM is $\sim 50 \mathrm{keV}$ around $10 \mathrm{MeV}$ energies. The relative energy spread was reduced becoming smaller than $3 \%$. In addition, one can see the increase of the detection signal on the axis up to 10 times for energy range near $12.4 \mathrm{MeV}$. This means that the pulse solenoid also works as a collecting lens for these bunches.

Typical beam images for five successive shots detected by the ESM after the energy selection arranged for electron energy of $12.4 \mathrm{MeV}$ by the solenoid are given in Fig. 7. The charging voltage and corresponding field strength on the axis of the pulse solenoid for this electron energy were $V_{0}=$ $650 \mathrm{~V}$ and $B_{z}=0.74 \mathrm{~T}$, respectively. In Fig. 7, horizontal and vertical axes correspond to energy and radial distribution of the beams respectively. Though the intensity of the spots were fluctuating, as seen in Fig. 7, the central position of the beams in the vertical axis and beam energy on the horizontal axis are stable and repeatable. Although it is difficult to estimate beam spot size with phosphor screen images due to its blurring, the upper limit of beam spot sizes was evaluated to be less than $1.6 \mathrm{~mm}$ in FWHM. Stability of the energy spectra is approximately better than $\pm 25 \mathrm{keV}$. The pointing stability of the beams is approximately $0.3 \mathrm{mrad}$, the same as the one shown in Fig. 5. We attribute the fluctuation of the emission, which corresponds to beam charge at the EMS screen, to fluctuation of electron beams produced by LWFA.

Energy selectability was characterized in the experiment by changing the solenoid drive current. Typical ESM screen images and calibrated energy spectra for energy selected beams are given in Figs. 8(a) and 8(b). Beams, having initially thermal-like energy profiles, were selectively transported to the image screen of the ESM located at $2 \mathrm{~m}$ from the gas jet (T), while energy selectability for beams with energy from 10 to $25 \mathrm{MeV}$ were almost the same: $\Delta \varepsilon / \varepsilon \sim 2 \%$ to $3 \%$. For lower energies $\sim 2 \mathrm{MeV}$ and higher energies $\sim 35 \mathrm{MeV}$, the energy spreads were $5 \%$ and $1 \%$, respectively. In case of lower electron energy, the broader energy spectra are a result of a larger geometrical emittance (see Table I). In the case of $35 \mathrm{MeV}$ energy, an essential decrease of the number of electrons in this energy range is reflected. Detection efficiency of electrons with energy below $10 \mathrm{MeV}$ is relatively smaller than that for higher energy due to edge effects of the dipole magnets in the ESM. This results in the relatively smaller number of lower energy electrons $\sim 2 \mathrm{MeV}$.

The charge of bunches was estimated by the conventional technique [27] with use of electron multiplying CCDs (Princeton Instruments, EMCCD Pro-HS1KBX3 [28], chip size $13.3 \mathrm{~mm} \times 13.3 \mathrm{~mm}$ ) and the phosphor screen. The EMCCD chip was set behind the screen at $600 \mathrm{~mm}$; the solid angle was $\sim 3.9 \times 10^{-5} \mathrm{sr}$. The results of the measurements are summarized in Table I. The maximal charge of $1.6 \mathrm{pC}$ was generated for electrons with the energy $12.4 \mathrm{MeV}$. Again, the system was optimized for this electron energy and there was no optimization of the charge of thermal electrons. The use of an extra filtering, such as an aperture [(A) in Fig. 1] with a diameter of $500 \mu \mathrm{m}$ set in $\sim 101 \mathrm{~cm}$ away from the target (T), results in narrower, by a factor of 1.5 , energy spread, $\Delta \varepsilon / \varepsilon=1.9 \%$, of electron bunches as seen in Fig. 9. However, the bunch charge decreases stronger by a factor of 2.9 .

For the present laser and gas-jet parameters such as intensity, duration, prepulses, and density distribution, the total charge of accelerated electrons with thermal energy

TABLE I. The parameters for the energy selected electron beams by the pulse-driven solenoid. The bunch charges were estimated with the scintillation signals of the phosphor screen (DRZ-High [24]).

\begin{tabular}{|c|c|c|c|c|c|c|}
\hline Energy $(\mathrm{MeV})$ & 2.08 & 8.50 & 12.4 & 18.3 & 26.4 & 34.7 \\
\hline Transvers geometrical emittance (mm-mrad) & $8.1 \times 10^{-3}$ & $5.9 \times 10^{-3}$ & $4.8 \times 10^{-3}$ & $3.9 \times 10^{-3}$ & $3.8 \times 10^{-3}$ & $2.8 \times 10^{-3}$ \\
\hline Scintillation signal (photons/sr) & $2.57 \times 10^{9}$ & $2.64 \times 10^{9}$ & $5.46 \times 10^{9}$ & $2.38 \times 10^{9}$ & $1.29 \times 10^{9}$ & $6.02 \times 10^{8}$ \\
\hline Corresponding charge $(\mathrm{pC})$ & 0.370 & 0.750 & 1.600 & 0.680 & 0.370 & 0.170 \\
\hline
\end{tabular}


(a)

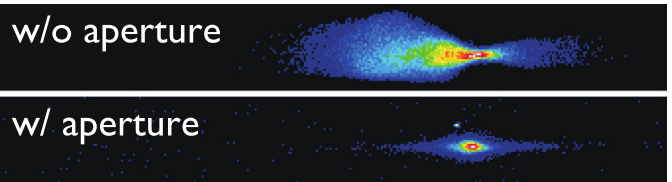

(b)

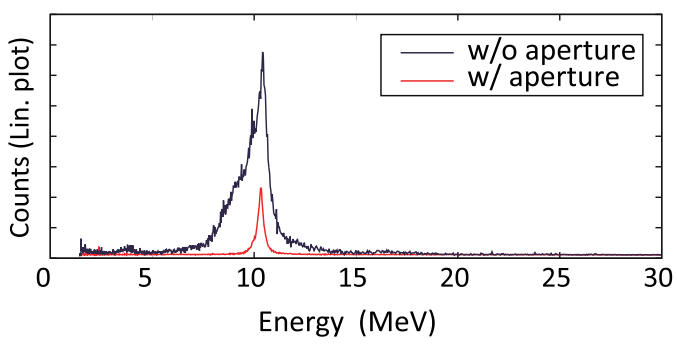

FIG. 9. Typical images of electron beams on an ESM screen with and without $500 \mu \mathrm{m}$ aperture (a), and the corresponding energy spectra of the electron beams on the axis (b).

distribution measured before energy selection by the solenoid was not high, about 120 pC. Optimization of the total charge of these electrons was out of the scope of this work [29].

Presently such well-defined beams can be a useful tool to probe different regimes in the staged LWFA. In order to test their feasibility for staging experiments, we performed a preliminary $2 \mathrm{D}$ particle in cell simulation. In the simulation the electron beam is assumed to be emitted from the cathode (injector stage). Then this electron beam is coupled with the booster wake field generated by another laser

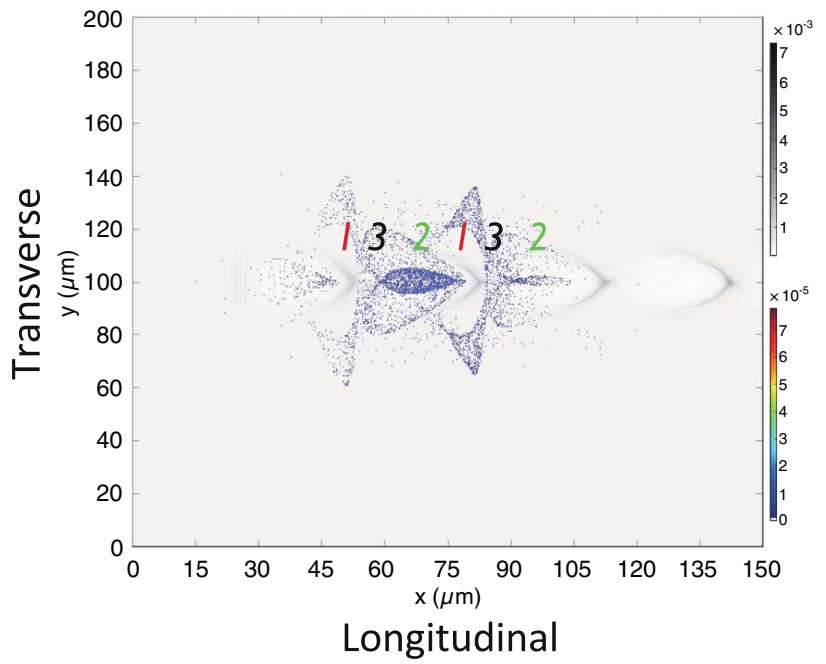

FIG. 10. Density distribution of plasma and beam electrons in the booster wake field; initial beam parameters are as follows: Bunch charge is $10 \mathrm{pC}$, diameter is $40 \mu \mathrm{m}$ and length is $80 \mu \mathrm{m}$, energy is $50 \mathrm{MeV}$ and $\Delta \varepsilon / \varepsilon=2 \%$. Plasma density in the booster is $1 \times 10^{18} \mathrm{~cm}^{-3}$; the laser pulse has $25 \mathrm{fs}$ duration and intensity $I=1 \times 10^{19} \mathrm{~W} / \mathrm{cm}^{2}$ and is focused to $25 \mu \mathrm{m}$ diameter. (1) is acceleration zone, (2) is deceleration zone, (3) is phase rotation zone. Beam electrons are marked by blue and plasma density is given in black.

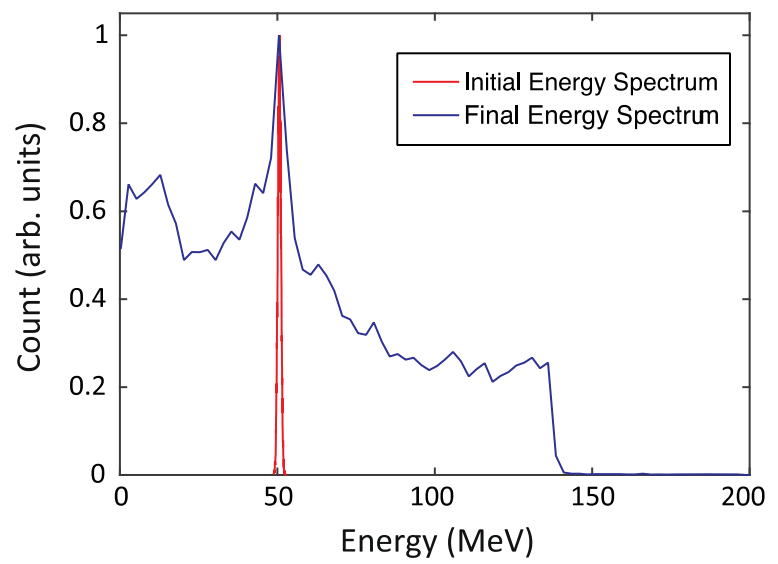

FIG. 11. Typical energy distribution of beam electrons at $t=$ 3.3 ps for parameters given in Fig. 10.

pulse. Here, we briefly present the result of beam evolution. We chose a long and spatially broad beam to probe numerically the interaction of a narrow band electron beam with a laser pulse wake in different regimes excluding dependency on the injection phase. In Figs. 10 and 11 typical parameters of such an interaction are shown. One can clearly separate three possible scenarios of interaction: Acceleration (marked by 1), deceleration (2), and phase rotation (3). Evolution of electron beams strongly depends on beam sizes and injection alignment as well as on the laser pulse energy. Characterization of electron injection for acceleration, or for deceleration, or for phase-rotation particularly is out of the scope of this paper and will be presented later.

\section{CONCLUSION}

In conclusion, we have produced and characterized the energy selectable $\mathrm{MeV}$ class of the plasma cathodes based on LWFA combined with the beam energy filtering technique. Electron beams extracted from the cathode may serve as an efficient injector in multistage LWFA technique as well as for ultrafast electron imaging.

In the experiment, electron beams with energy from 2 up to $35 \mathrm{MeV}$ and charges $\sim 1 \mathrm{pC}$ were focused and transported along $2 \mathrm{~m}$ with energy spread $\Delta \varepsilon / \varepsilon$ below $3 \%$ and with spot size smaller than 1 mrad in FWHM, respectively. Moreover, in the case of moderate energies from 10 to $25 \mathrm{MeV}$ the pulse solenoid was also a good collector providing an increase of the number of electrons on the axis at the image screen more than 10 times. The relatively small size of selected beams in a long distance allows the use of such beams as injectors for the second stage of multistage LWFA with geometrical efficiency $\sim 1 \%$ only without applying additional focusing techniques. The beam size is also important for further injection of the beams to the acceleration wakefield in the second stage. Since the maximal available size of a laser beam providing $a_{0} \sim 1$ is within $\sim 100 \mu \mathrm{m}$ the injection efficiency is $\sim 1 \%$ 
geometrically for the present parameters. A way for increasing this efficiency is in (i) tightly focusing the beams while shortening the distance between the plasma cathode and a booster (the second stage) via increasing drive currents of solenoids or (ii) use of extra plasma lens, for example as in Ref. [30].

Although further measurements for characterization of beam parameters such as bunch durations have yet to be done, the demonstrated narrow-band energy beams are a serious step forward in the creation of the staging LWFA and elaboration of future ultrafast electron beam diffraction imaging diagnostics.

\section{ACKNOWLEDGMENTS}

This work was supported and funded by the ImPACT Program of Council for Science, Technology and Innovation (Cabinet Office, Government of Japan). Part of this work was also supported by the JST-MIRAI Program Grant No. JPMJMI17A1. Also, we appreciate Dr. M. Yoshida, Dr. T. Natsui, and Dr. K. Kawakami at KEK, Japan, for their support on the pulse driver construction.

[1] T. Tajima and J. M. Dawson Laser Electron Accelerator, Phys. Rev. Lett. 43, 267 (1979).

[2] S. P. D. Mangles, C. D. Murphy, Z. Najmudin, A. G. R. Thomas, J. L. Collier, A. E. Dangor, E. J. Divall, P. S. Foster, J. G. Gallacher, C. J. Hooker, D. A. Jaroszynski, A. J. Langley, W. B. Mori, P. A. Norreys, F. S. Tsung, R. Viskup, B. R. Walton, and K. Krushelnick, Monoenergetic beams of relativistic electrons from intense laserplasma interactions, Nature (London) 431, 535 (2004).

[3] C. G. R. Geddes, Cs. Toth, J. van Tilborg, E. Esarey, C. B. Schroeder, D. Bruhwiler, C. Nieter, J. Cary, and W. P. Leemans, High-quality electron beams from a laser wakefield accelerator using plasma-channel guiding, Nature (London) 431, 538 (2004).

[4] J. Faure, Y. Glinec, A. Pukhov, S. Kiselev, S. Gordienko, E. Lefebvre, J.-P. Rousseau, F. Burgy, and V. Malka, A laser-plasma accelerator producing monoenergetic electron beams, Nature (London) 431, 541 (2004).

[5] P. Gibbon, Short Pulse Laser Interaction with Matter (World Scientific, Singapore, 2005).

[6] W. P. Leemans, A. J. Gonsalves, H. S. Mao, K. Nakamura, C. Benedetti, C. B. Schroeder, Cs. Toth, J. Daniels, D. E. Mittelberger, S. S. Bulanov, J. L. Vay, C. G. R. Geddes, and E. Esarey, Multi-GeV Electron Beams from CapillaryDischarge-Guided Subpetawatt Laser Pulses in the SelfTrapping Regime, Phys. Rev. Lett. 113, 245002 (2014).

[7] S. Tokita, M. Hashida, S. Inoue, T. Nishoji, K. Otani, and S. Sakabe, Single-Shot Femtosecond Electron Diffraction with Laser-Accelerated Electrons: Experimental Demonstration of Electron Pulse Compression, Phys. Rev. Lett. 105, 215004 (2010).

[8] Z. H. He, A. G. R. Thomas, B. Beaurepaire, J. A. Nees, B. Hou, V. Malka, K. Krushelnick, and J. Faure, Electron diffraction using ultrafast electron bunches from a laserwakefield accelerator at $\mathrm{kHz}$ repetition rate, Appl. Phys. Lett. 102, 064104 (2013).

[9] J. M. Cole, J. C. Wood, N. C. Lopes, K. Poder, R. L. Abel, S. Alatabi, J. S. J. Bryant, A. Jin, S. Kneip, K. Mecseki, D. R. Symes, S. P. D. Mangles, and Z. Najmudin, Laserwakefeld accelerators as hard X-ray sources for 3D medical imaging of human bone, Sci. Rep. 5, 13244 (2015).

[10] A. Buck, J. Wenz, J. Xu, K. Khrennikov, K. Schmid, M. Heigoldt, J. M. Mikhailova, M. Geissler, B. Shen, F. Krausz, S. Karsch, and L. Veisz, Shock-Front Injector for High-Quality Laser-Plasma Acceleration, Phys. Rev. Lett. 110, 185006 (2013).

[11] J. Faure, Y. Glinec, J. J. Santos, F. Ewald, J.-P. Rousseau, S. Kiselev, A. Pukhov, T. Hosokai, and V. Malka, Observation of Laser-Pulse Shortening in Nonlinear Plasma Waves, Phys. Rev. Lett. 95, 205003 (2005).

[12] A. Giulietti, A. André, S. Dobosz Dufrénoy, D. Giulietti, T. Hosokai et al., Space- and time-resolved observation of extreme laser frequency upshifting during ultrafastionization, Phys. Plasmas 20, 082307 (2013).

[13] T. Hosokai, M. Kando, H. Dewa, H. Kotaki, S. Kondo, N. Hasegawa, K. Nakajima, and K. Horioka, Optical guidance of terrawatt laser pulses by the implosion phase of a fast Z-pinch discharge in a gas-filled capillary, Opt. Lett. 25, 10 (2000).

[14] A. Butler, D. J. Spence, and S. M. Hooker, Guiding of High-Intensity Laser Pulses with a Hydrogen-Filled Capillary Discharge Waveguide, Phys. Rev. Lett. 89, 185003 (2002).

[15] W. P. Leemans, B. Nagler, A. J. Gonsalves, Cs. Tóth, K. Nakamura, C. G. R. Geddes, E. Esarrey, C. B. Schroeder, and S. M. Hooker, GeV electron beams from a centimetrescale accelerator, Nat. Phys. 2, 696 (2006).

[16] T. Hosokai, K. Kinoshita, A. Zhidkov, A. Maekawa, A. Yamazaki, and M. Uesaka, Effect of External Static Magnetic Field on the Emittance and Total Charge of Electron Beams Generated by Laser-Wakefield Acceleration, Phys. Rev. Lett. 97, 075004 (2006).

[17] N. Nakanii, T. Hosokai, K. Iwasa, S. Masuda, A. Zhidkov, N. Pathak, H. Nakahara, Y. Mizuta, N. Takeguchi, and R. Kodama, Transient magnetized plasma as an optical element for high power laser pulses, Phys. Rev. ST Accel. Beams 18, 021303 (2015).

[18] G. Golovin, S. Banerjee, S. Chen, N. Powers, C. Liu, W. Yan, J. Zhang, P. Zhang, B. Zhao, and D. Umstadter, Control and optimization of a staged laser-wakefield accelerator, Nucl. Instrum. Methods Phys. Res., Sect. A 830, 375 (2016).

[19] E. Esarey, C. B. Schroeder, and W. P. Leemans, Physics of laser-driven plasma-based electron accelerators, Rev. Mod. Phys. 81, 1229 (2009).

[20] T. Ohkubo, S. V. Bulanov, A. G. Zhidkov, T. Esirkepov, J. Koga, M. Uesaka, and T. Tajima, Wave-breaking injection of electrons to a laser wake field in plasma channels at the strong focusing regime, Phys. Plasmas 13, 103101 (2006).

[21] T. Hosokai, K. Kinoshita, T. Ohkubo, A. Maekawa, M. Uesaka, A. G. Zhidkov, A. Yamazaki, H. Kotaki, M. Kando, K. Nakajima, S. V. Bulanov, P. Tomassini, A. Giulietti, and D. Giulietti, Observation of strong 
correlation between quasimonoenergetic electron beam generation by laser wakefield and laser guiding inside a preplasma cavity, Phys. Rev. E 73, 036407 (2006).

[22] S. P. Weathersby et al., Mega-electron-volt ultrafast electron diffraction at SLAC National Accelerator Laboratory, Rev. Sci. Instrum. 86, 073702 (2015).

[23] A. E. Siegman, Lasers (University Science Books, Mill Valley, CA, 1986).

[24] DRZ-High, (the phosphor screen), https://www .m-chemical.co.jp/en/products/departments/mcc/ledmat/ product1201037_7550.html.

[25] Triple insulated wire TEX-E, https://www.furukawa.co.jp/ tex-e/en/product/texe_series.html.

[26] Power IGBT CM900HB-90H, (the solid-state switching device), http://www.mitsubishielectric.co.jp/semiconductors/ content/product/powermod/powmod/hvigbtmod/hvigbt/ cm900hb-90h_j.pdf.
[27] A. Buck, K. Zeil, A. Popp, K. Schmid, A. Jochmann, S. D. Kraft, B. Hidding, T. Kudyakov, C. M. S. Sears, L. Veisz, S. Karsch, J. Pawelke, R. Sauerbrey, T. Cowan, F. Krausz, and U. Schramm, Absolute charge calibration of scintillating screens for relativistic electron detection, Rev. Sci. Instrum. 81, 033301 (2010).

[28] EMCCD Pro-HS1KBX3, https://www.princetonin struments.com/userfiles/files/assetLibrary/Datasheets/ ProEM-HS1KBX3-10um-and-1024K-BX3-datasheet-P45-16-17.pdf.

[29] M. Kando et al., Electron acceleration by a nonlinear wakefield generated by ultrashort (23-fs) high-peak-power laser pulses in plasma, Phys. Rev. E 71, 015403 (2005).

[30] S. Steinke, J. V. Tilborg, C. Benedetti, C. G. R. Geddes, C. B. Schroeder, J. Daniels, K. K. Swanson, A. J. Gonsalves, K. Nakamura, N. H. Matlis, B. H. Shaw, E. Esarey, and W. P. Leemans, Multistage coupling of independent laser-plasma accelerators, Nature (London) 530, 190 (2016). 